\title{
An immunohistochemical, clinical and electroneuromyographic correlative study of the neural markers in the neuritic form of leprosy
}

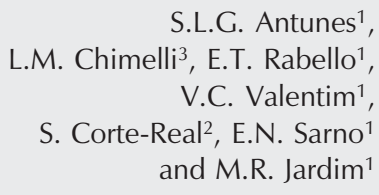

S.L.G. Antunes ${ }^{1}$,

L.M. Chimelli ${ }^{3}$, E.T. Rabello ${ }^{1}$, V.C. Valentim ${ }^{1}$,

S. Corte-Real ${ }^{2}$, E.N. Sarno ${ }^{1}$ and M.R. Jardim ${ }^{1}$

\author{
${ }^{1}$ Laboratório de Hanseníase, ²Departamento de Ultra-estrutura e Biologia Celular, \\ Fundação Oswaldo Cruz, Rio de Janeiro, RJ, Brasil \\ ${ }^{3}$ Departamento de Patologia, Universidade Federal do Rio de Janeiro, \\ Rio de Janeiro, RJ, Brasil
}

\section{Correspondence}

S.L.G. Antunes

Laboratório de Hanseníase

Fundação Oswaldo Cruz

Av. Brasil, 4365

21045-900 Rio de Janeiro, RJ

Brasil

E-mail: santunes@fiocruz.br

Research supported by FAPERJ and POM funds of the FIOCRUZ.

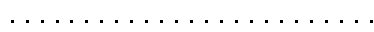

Received November 28, 2005 Accepted April 20, 2006

\begin{abstract}
The nerve biopsies of 11 patients with pure neuritic leprosy were submitted to routine diagnostic procedures and immunoperoxidase staining with antibodies against axonal (neurofilament, nerve growth factor receptor (NGFr), and protein gene product (PGP) 9.5) and Schwann cell (myelin basic protein, S-100 protein, and NGFr) markers. Two pairs of non-adjacent histological cross-sections of the peripheral nerve were removed for quantification. All the fascicles of the nerve were examined with a 10X-ocular and 40X-objective lens. The immunohistochemistry results were compared to the results of semithin section analysis and clinical and electroneuromyographic data. Neurofilament staining was reduced in $100 \%$ of the neuritic biopsies. NGFr positivity was also reduced in $81.8 \%$, PGP staining in $100 \%$ of the affected nerves, S100 positivity in $90.9 \%$, and myelin basic protein immunoreactivity in $90.9 \%$. Hypoesthesia was associated with decreased NGFr (81.8\%) and PGP staining (90.9\%). Reduced potential amplitudes (electroneuromyographic data) were found to be associated with reduced PGP $9.5(63.6 \%)$ and nerve fiber neurofilament staining $(45.4 \%)$ by immunohistochemistry and with loss of myelinated fibers $(100 \%)$ by semithin section analysis. On the other hand, the small fibers (immunoreactive dots) seen amid inflammatory cells continued to be present even after $40 \%$ of the larger myelinated fibers had disappeared. The present study shows an indepth view of the destructive effects of leprosy upon the expression of neural markers and the integrity of nerve fiber. The association of these structural changes with the clinical and electroneuromyographic manifestations of leprosy peripheral neuropathy was also discussed.
\end{abstract}

\section{Introduction}

Leprosy is primarily a peripheral nerve disease despite prominent involvement of the skin in a variety of cutaneous lesions and
Key words

- Neurofilament protein

- Nerve growth factor receptor

- S-100 protein

- Myelin basic protein

- Gene product 9.5

- Neuritic leprosy

- Peripheral neuropathy damage to the upper respiratory tract, eyes, and viscera (1). Pure neuritic leprosy, however, is a form of the disease in which, except for hypoesthesia, no cutaneous manifestations are found. In this respect, the risk of 
developing a disability due to leprosy is directly related to the presence of Mycobacterium leprae in the nerves and to the nerve damage detected on the occasion of the first patient visit (2). On average, neuritic leprosy accounts for about $10 \%$ of all leprosy cases and is particularly difficult to diagnose if acid-fast bacilli are not found in the skin smears or histological sections of the nerves (2-5). In this regard, PCR has proven to be very useful in confirming a diagnosis of pure neuritic leprosy (6).

The peripheral neuropathy occurring in leprosy is an inflammatory disease of the nerves. The inflammatory infiltrate of the affected nerves may be composed of either a mature epithelioid granuloma or of acid-fast bacilli-loaded macrophages exhibiting foamy changes $(7,8)$. The pathogenesis of leprosy neuropathy is far from being fully understood, especially with respect to the mechanisms involved in nerve fiber lesions. In addition, axonal loss accompanied by Wallerian degeneration is observed at the end stage of leprosy neuropathy $(8,9)$, and T-cell cytotoxic activity on Schwann cells (Sc) has been demonstrated in vitro (10). Axonal degeneration following Sc death, demyelination, and the environmental changes induced by this specific inflammatory process could be the pathogenic mechanism responsible for nerve function impairment. Demyelination, best detected by the teasing of axonal segments, is also found in leprosy (11). In 2002, for example, Rambukkana et al. (12) reported that $M$. leprae had a strong demyelinating effect on infected Sc neuron cultures and Rag-1 knockout mice.

The inflammatory process undeniably plays a crucial role in the destruction of nerve fibers. However, the molecular mechanisms leading to axonal degeneration and/or demyelination in leprosy have rarely been explored in the literature. Thus, in the present study, we investigated the expression of axonal and Sc markers in nerve biopsies taken from 11 pure neuritic leprosy patients.
An attempt was made to characterize the specific pattern of change in leprosy neuropathy and to describe the modifications in molecular expression within the leprosy-affected nerves. Immunoperoxidase staining of the axonal neurofilament protein (13) and protein gene product (PGP) 9.5 (14) was carried out. Sc S-100, nerve growth factor receptor (NGFr) $(15,16)$, and myelin basic protein (17) were also employed for immunostaining. The results of immunohistochemistry were then correlated with the patients' clinical, electroneurophysiologic, and histological data.

The purpose of this study was to search for correlations between nerve fiber status in pure neuritic leprosy and the clinical and electroneuromyographic presentation of the disease.

\section{Material and Methods}

\section{Patient selection and routine biopsy procedures}

The nerve biopsies of 11 patients with pure neuritic leprosy were submitted to routine histopathological analysis. Biopsies were collected in the Leprosy Outpatient Clinic of the Department of Tropical Medicine, Oswaldo Cruz Foundation, Rio de Janeiro, RJ, Brazil. The biopsies were fixed in Carson's fixative solution (Millonig buffered formalin) (18), embedded in paraffin and 4- $\mu \mathrm{m}-$ thick sections were stained with hematoxylin-eosin, Gomori's trichrome, and Wade staining.

A diagnosis of neuritic leprosy was confirmed on the basis of criteria elaborated in our laboratory (6). Briefly, the diagnosis of neuritic leprosy can be based on the following parameters 1) defined diagnosis: patients have a clinical presentation consistent with leprosy, with histopathological examinations demonstrating an inflammatory infiltrate with acid-fast bacilli, and $M$. leprae-specific DNA detected by PCR and serum anti-phenolic 
glycolipid-1 (PGL-1) levels, and 2) probable diagnosis: patients have a clinical presentation consistent with leprosy, with the presence of epithelioid granulomatous neuritis, mononuclear cell endoneuritis and fibrosis. In this case, while a diagnosis of leprosy is not defined by a golden test, there is a high probability (over 99\%) of it being confirmed if the case is from Brazil, where leprosy is a highly prevalent cause of peripheral neuropathy.

\section{Serum PGL-1 determination}

Detection of serum anti-PGL-1 antibody (19) and of M. leprae DNA by PCR (20) was carried out in order to provide an additional parameter for a leprosy diagnosis. NT-PBSA was used as the semi-synthetic analogue of the PGL-1 antigen. ELISA was performed essentially as described (19) except for the following modifications: serum dilution was 1:500, the conjugate dilution was $1: 10,000$, and the substrate used was $0.04 \%$ tetramethylbenzine and $0.04 \%$ urea peroxide in $0.1 \mathrm{M}$ sodium acetate citric acid buffer, $\mathrm{pH}$ 4.0. The cut-off value used for positivity was the optical density (OD) at $450 \mathrm{~nm}=0.2$. In order to control for plate-toplate and day-to-day variation, a positive reference serum was included in triplicate on each plate. The color reactions of the entire plate were stopped with $50 \mu \mathrm{L} 2 \mathrm{~N}$ $\mathrm{H}_{2} \mathrm{SO}_{4}$ when the OD at $492 \mathrm{~nm}$ from a positive control serum reached a value of 0.6 . The ODs were measured in a spectrophotometer using a 492-nm filter. All sera were tested in duplicate and the ELISA results are reported as mean absorbance of the duplicates. The final OD value of each serum sample was calculated by subtracting the OD value of wells coated with BSA from the OD value of the test wells coated with DBSA.

\section{Immunohistochemistry}

Immunoperoxidase staining with anti- bodies against neural markers of axons (neurofilament (NF), NGFr, and PGP 9.5) and Sc (myelin basic protein, S-100 protein, and NGFr) was performed on paraffin-embedded sections. Two biopsies with normal histological appearance taken from patients with symptoms of neuropathy were used as controls. Only pure neuritic leprosy patients were selected for this study since the above procedures are only ethically warranted when it is necessary to confirm a leprosy diagnosis in patients without cutaneous lesions.

Some of the routine paraffin-embedded sections were stained with the avidin-biotin complex and submitted to immunoperoxidase staining with streptavidin-biotin-peroxidase (Dako A/S, Glostrup, Denmark). Primary antibodies were mouse anti-NGFr (Dako Corp., Carpinteria, CA, USA, 1/50), rabbit anti-PGP 9.5 (UltraClone, Cambridge, England, 1/400), rabbit anti-S100 (Dako Corp., 1/100), mouse anti-NF200 protein (Sigma, St. Louis, MO, USA, 1/400), and rabbit anti-myelin basic protein (Dako Corp., 1/200) diluted in Trisbuffered saline, pH 7.4 (Sigma). Immunostaining of the sections was developed in a solution containing diaminobenzidine (Sigma) and hydrogen peroxide. The sections were placed on slides, counterstained with Meyer's hematoxylin, dehydrated, and mounted with Entellan medium (Merck, Darmstadt, Germany).

Control sections with non-immune serum from the same animal of the second antibody (Dako Corp.) and another slide with only droppings of diaminobenzidine (Sigma) were utilized as specificity and endogenous peroxidase controls, respectively. Paraffin-embedded sections of skin, which are S-100-, NF-, NGFr, PGP 9.5-positive, were also used in order to control processing of the tissues (internal controls). The immunostaining results were correlated with the semithin section analyses and clinical and electroneuromyographic data. Immunoreactivity was scored from $0=$ no staining to $4=$ maximal staining. 


\section{Semithin section processing}

A small fragment of the nerve was processed to obtain semithin sections which were fixed with $2.5 \%$ glutaraldehyde, rinsed with $0.1 \mathrm{M}$ sodium cacodylate, postfixed with $2 \%$ osmium tetroxide, dehydrated with acetone, embedded in epon (21), and sectioned with a Leica (Leica Microsystems, Wetzlar, Germany) ultramicrotome. The 0.5to $1-\mu \mathrm{m}$-thick sections were stained with Toluidine blue, examined with a Nikon E400 Eclipse light microscope (Kawasaki, Tanagawa, Japan) and documented with a Cool Snap digital camera (Image Processing Solutions, North Reading, MA, USA). The images were then saved in a tagged-image file format and printed on glossy paper with an HP deskjet 640 printer (Loveland, CO, USA). Fiber loss and staining intensity were estimated semi-quantitatively using a score ranging from $0=$ total loss to $4=$ normal fiber density (see Table 1).

The changes detected in the present study reflected differences between the biopsies from leprosy patients and from controls. No follow-up assessment was conducted.

\section{Results}

The average age $( \pm \mathrm{SD})$ of the neuritic leprosy patients (10 males and 1 female) studied was $42.9 \pm 18.09$ years. None of the patients had previously received any specific anti-leprosy drugs. Sensory impairment and paresthesia were found in all 11 patients $(100 \%)$, while nerve enlargement was detected in $8(72.7 \%)$, nerve pain in $3(27.2 \%)$, and paresis in $10(90.9 \%)$. The amplitude of the axonal potential was reduced in 7 of the biopsied nerves $(63.6 \%)$, conduction velocity was reduced in 10 (90.9\%), and disturbances in potential conduction and amplitude were detected in 7 (63.6\%). In addition, 10 patients $(90.9 \%)$ had multiplex mononeuropathy and one had polyneuropathy. Table 1 lists the neurological alterations found in the biopsied nerves together with the results of the electroneuromyography test.

Histological examination of the nerves showed variations in the spread of the inflammatory infiltrate, which was related to the number of nerve fibers still present in the nerve fascicles. The intensity and distribution of the immunoreactive signals varied according to the number of fibers and the proportion of the endoneurium occupied by inflammatory cells.

Immunohistochemical staining of small and large myelinated fibers (see Figures 1 and 2, and Table 2) and of small immunoreactive clustered dots (Figures 1B, 2A and 2D) surrounded by the cytoplasm of nucleated cells was observed. These dots corresponded to a cross-section of the remaining small fibers (some of them presumably nonmyelinated), which were preserved despite occupation of the endoneurium by the inflammatory infiltrate.

A description of the staining with each marker follows: 1) NF200 protein produced the strongest axoplasm staining in nerve fibers. Neuritic biopsies had a lower score with respect to both large and small fibers in $100 \%$ of the biopsies (Figure 1A). 2) S-100 positivity of both large (myelinated) and small fibers was reduced in $90.9 \%$ of the biopsies. Sometimes irregular and exhibiting a granular pattern in small fibers (Figure 1B), the S-100-positive pattern appeared as an immunoreactive cytoplasmic rim on the outer surface of myelinated fibers (Figure 1C) and in the cytoplasm of non-myelinating Sc (Figure 1B). S100-positive Sc were found in the endoneurial compartment of fascicles infiltrated by inflammatory cells where no staining of the axonal components was observed. Despite some apparent similarities, S100 and myelin basic protein (MBP) staining of myelinated fibers showed distinct patterns: anti-MBP did not stain the cytoplasm of Sc surrounding myelinated fibers but only their myelin sheath (Figure 1D). On the other hand, anti-S100 was found to stain the 
abaxonal or adaxonal rim of the Sc cytoplasm (Figure 1D). 3) Fiber MBP immunoreactivity was decreased in $90.9 \%$ of the biopsies (Figure 2C). The final score depended on the number of endoneurial fibers present. Therefore, staining was restricted to the myelinated fibers not affected by the inflammatory invasion (Figure 1D). MBP negativity (Figure 2C) concomitant with PGP 9.5 (Figure 2B) and NGFr positivity (Figure 2D) indicated that in $40 \%$ of the biopsies small fibers remained in the endoneurial compartment despite the absence of $m$ fibers. 4) The NGFr expressed by both axons and their surrounding non-myelinating $\mathrm{Sc}$ showed reduced staining in $81.8 \%$ of the biopsies compared to control nerves. NGFrpositive Sc were seen amid inflammatory cells whose staining pattern was similar to that obtained with S100 staining (compare Figures $1 \mathrm{~B}$ and $2 \mathrm{~A}$ ). It was not possible to distinguish small NGFr-positive axons from NGFr-positive Sc since both structures emitted positive signals on the non-myelinated fiber profiles that were in very close proximity to each other (Figure 2A and D). 5) PGP 9.5 staining was similar to NF200 immunoreactivity and was reduced in $100 \%$ of the leprosy biopsies (compare Figures 1A and
2B). Both small and large PGP-positive fibers could also be seen (Figure 2B).

The nerve biopsies of the controls displayed a normal histological appearance and a maximal staining grade. In addition, alterations such as a reduced number of myelinated fibers and axonal degeneration, regeneration (sprouting), and remyelination were completely absent (score $=0$ ) on semithin sections of control biopsies (see Table 2).

Semithin section analysis showed a reduced number of myelinated fibers in $100 \%$ of the lepromatous biopsies (Figure 3A-D). A higher number of this type of fiber was seen when the infiltrate was lepromatous (Figure $3 \mathrm{~A}$ and $\mathrm{D}$ ). Axonal degeneration was present in $10 \%$ of the biopsies, axonal regeneration in $30 \%$, and axonal remyelination in $20 \%$ even though these three morphological alterations were inconspicuous. In 6 biopsies (54.5\%) the endoneurial space, completely devoid of myelinated fibers, was occupied either by lepromatous infiltrates with acid-fast bacilli-loaded vacuolated macrophages (Figure 3C) or by mononuclear cells (Figure 3B), which in some cases formed tuberculoid granulomas (Table 2).

Hypoesthesia was associated with a decrease in NGFr and PGP staining in 81.8 and

Table 1. Clinical and electroneuromyographic characteristics of the leprosy patients studied.

\begin{tabular}{|c|c|c|c|c|c|c|c|c|}
\hline \multirow{2}{*}{$\begin{array}{l}\text { Patient } \\
\text { No. }\end{array}$} & \multirow{2}{*}{$\begin{array}{c}\text { Nerve } \\
\text { pain }\end{array}$} & \multirow{2}{*}{$\begin{array}{l}\text { Sensory } \\
\text { impairment }\end{array}$} & \multirow[t]{2}{*}{ Paresthesia } & \multirow{2}{*}{$\begin{array}{c}\text { Motor } \\
\text { impairment }\end{array}$} & \multicolumn{3}{|c|}{ Neurophysiology } & \multirow{2}{*}{$\begin{array}{l}\text { Nerve } \\
\text { biopsy }\end{array}$} \\
\hline & & & & & Classification & $\begin{array}{l}\text { Number of nerve } \\
\text { impairments }\end{array}$ & $\begin{array}{l}\text { Conduction } \\
\text { of nerve biopsy }\end{array}$ & \\
\hline 1 & - & + & + & - & Combined & Multiplex mononeuropathy & Present & Right sural \\
\hline 2 & + & + & + & + & Demyelinating & Multiplex mononeuropathy & Absent & Right ulnar \\
\hline 3 & - & + & + & - & Combined & Multiplex mononeuropathy & Absent & Right ulnar \\
\hline 4 & - & + & + & - & Combined & Multiplex mononeuropathy & Absent & Left ulnar \\
\hline 5 & - & + & + & - & Demyelinating & Multiplex mononeuropathy & Absent & Right ulnar \\
\hline 6 & - & + & + & + & No classification & Polyneuropathy & Absent & Left sural \\
\hline 7 & - & + & + & - & Combined & Multiplex mononeuropathy & Absent & Left ulnar \\
\hline 8 & - & + & + & + & Combined & Multiplex mononeuropathy & Absent & Left ulnar \\
\hline 9 & + & + & + & + & Demyelinating & Multiplex mononeuropathy & Absent & Left ulnar \\
\hline 10 & - & + & + & + & Combined & Multiplex mononeuropathy & Absent & Right sural \\
\hline 11 & + & + & + & + & Combined & Multiplex mononeuropathy & Absent & Left ulnar \\
\hline
\end{tabular}

Combined $=$ concomitant presence of reduced neuropotential amplitude and reduced conduction velocity . 
Figure 1. Immunohistochemical staining of neurofilament (NF), S100 protein and comparison of S100 protein and myelin basic protein (MBP) stainings of myelinated fibers. $A, \mathrm{~A}$ few NF-positive nerve fibers in the endoneurial compartment (end) of a nerve fascicle from a neuritic leprosy patient. Immunoreactive axoplasm of large myelinated fibers (arrows) as well as smaller axons (arrowheads), not seen by routine staining, can be observed. Inflammatory cells are seen in this field. $B, \mathrm{~S}-100$-immunoreactive Schwann cells (Sc) in the endoneurial compartment (end) of a nerve fascicle of a neuritic leprosy patient. The S-100 immunoreactivity corresponds to the Sc of nonmyelinated fibers, or, possibly, denervated Sc. The cytoplasmic staining of these cells presumably corresponds to the cell processes by which numerous axons are wrapped (arrows). There is a striking loss of myelinated fibers in this fascicle. C, S-100 immunoreactiv-
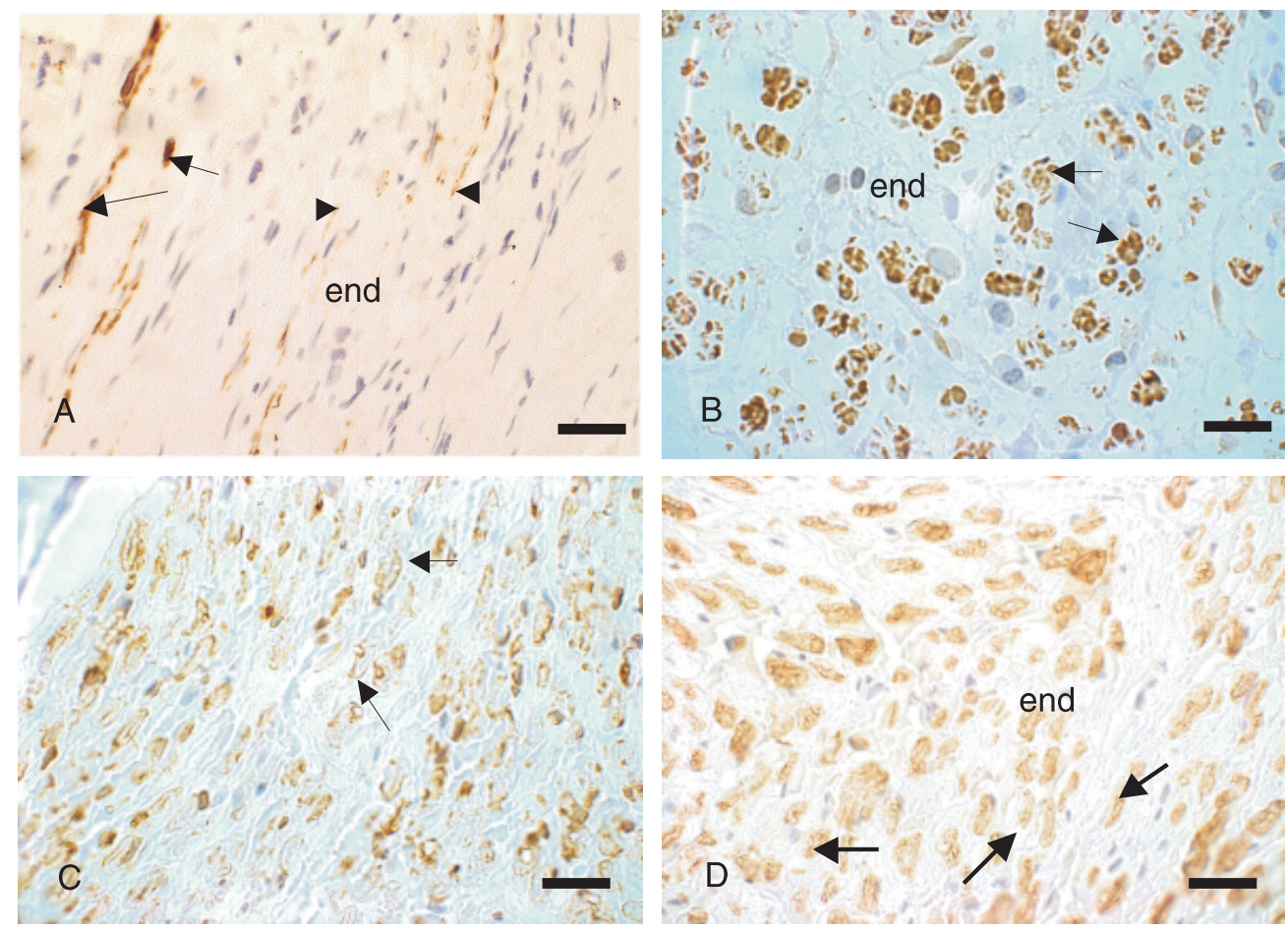

ity is quite similar to MBP staining but with some distinctive features of its own. The myelin sheath is weakly stained with S-100 antibody along with a stained outer rim corresponding to Sc abaxonal cytoplasm (arrows). D, The myelin sheath was more intensely stained by MBP antibody (arrows), and no rims of Sc cytoplasm were present. end $=$ endoneurium. Avidin-biotin immunoperoxidase and scale bar $=24 \mu \mathrm{m}$ for all panels.

Figure 2. Immunohistochemical staining of nerve growth factor receptor (NGFr), protein gene product 9.5 (PGP 9.5) and myelin basic protein (MBP). A, NGFr-immunoreactive $\mathrm{Sc}$ in the endoneurial compartment (end) of a nerve fascicle from a neuritic leprosy patient (cross-section). NGFr immunoreactivity (arrows) corresponds to the Sc of nonmyelinated fibers or, presumably, to denervated Sc (it is impossible to distinguish one from the other). The cytoplasmic heterogeneous NGFr staining of these cells at times overlaps with S-100 immunoreactivity. Some mononuclear cells (arrowheads) are also seen in the endoneurium. B, PGP 9.5-positive myelinated nerve fibers (arrows) in the endoneurial compartment (end) and small positive axons (thin arrows) can also be seen. The staining of this neuronal enzyme is not as strong as neurofilament staining (Figure 1A). C, Absence of MBP immunoreactivity
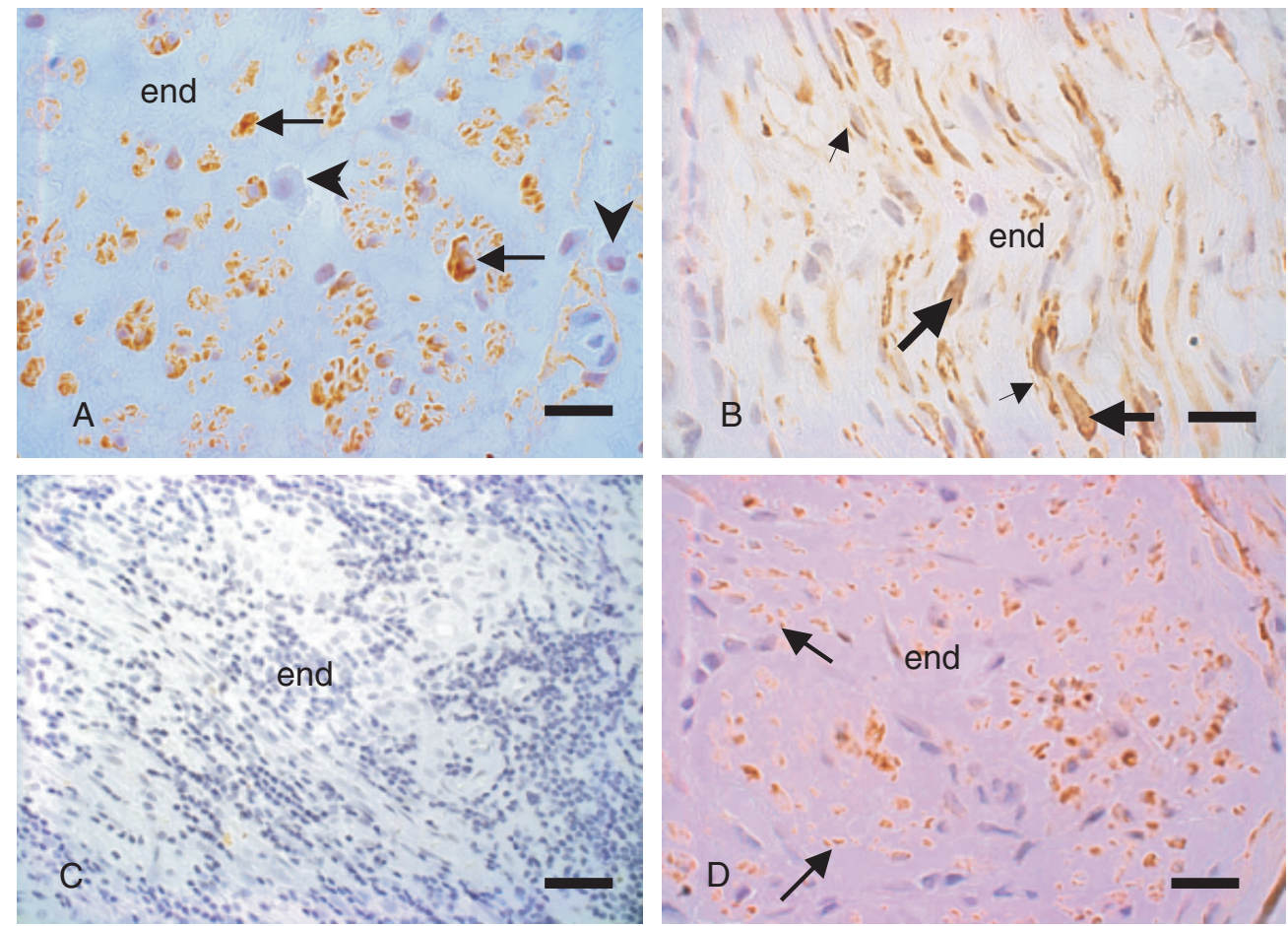

in the endoneurial compartment

(end) of a cross-section of a nerve fascicle taken from a neuritic leprosy patient. $D$, The same biopsy showed the presence of NGFr-positive nonmyelinated fibers (arrows) in the endoneurium (end). Avidin-biotin immunoperoxidase and scale bar $=24 \mu \mathrm{m}$ for all panels. 
Table 2. Laboratory, histopathological and immunohistochemical data of the leprosy patients studied.

\begin{tabular}{|c|c|c|c|c|c|c|c|c|c|c|c|c|}
\hline \multirow{2}{*}{$\begin{array}{l}\text { Patient } \\
\text { No. }\end{array}$} & \multirow[t]{2}{*}{ PCR } & \multirow[t]{2}{*}{ PGL-1 } & \multirow[t]{2}{*}{ Histopathology } & \multicolumn{4}{|c|}{ Examination of semithin sections ${ }^{a}$} & \multicolumn{5}{|c|}{ Immunoreactivity of nerve fibers ${ }^{b}$} \\
\hline & & & & Presence of myel fib & Ax deg & Ax reg & Ax rem & NF200 & NGFr & PGP & $S 100$ & MBP \\
\hline 1 & + & + & MNI, AFB 4+ & 0 & 0 & 0 & 0 & 0 & 2 & 1 & 3 & 0 \\
\hline 2 & + & + & LI, Fib, AFB 6+ & 0 & 0 & 0 & 0 & 0 & 0 & 1 & 0 & 0 \\
\hline 3 & + & - & Fib & 1 & 1 & 2 & 1 & ND & 0 & 0 & 0 & 1 \\
\hline 4 & - & - & TI, Fib & 0 & 0 & 2 & 0 & 0 & 0 & 0 & 3 & 1 \\
\hline 5 & ND & ND & TI, Fib & 1 & 0 & 0 & 0 & 1 & 2 & 1 & 3 & ND \\
\hline 6 & - & + & LI, TI, Fib, AFB 3+ & 1 & 0 & 0 & 0 & 0 & 0 & 0 & 0 & 0 \\
\hline 7 & - & - & MNI, Fib & ND & ND & ND & ND & 2 & 4 & 2 & 2 & 4 \\
\hline 8 & - & - & MNI & 3 & 0 & 1 & 1 & 1 & 0 & 1 & 3 & 3 \\
\hline 9 & - & ND & $\mathrm{TI}$ & 0 & 0 & 0 & 0 & 0 & 0 & 0 & 0 & 0 \\
\hline 10 & + & - & MNI, Fib & 0 & 0 & 0 & 0 & 0 & 4 & 3 & 4 & 0 \\
\hline 11 & - & - & TI, Fib & 0 & 0 & 0 & 0 & 2 & 3 & 3 & 0 & 0 \\
\hline
\end{tabular}

$\mathrm{AFB}=$ presence of acid-fast bacilli; $\mathrm{Ax}$ deg = axonal degeneration; $\mathrm{Ax}$ reg = axonal regeneration; $\mathrm{Ax}$ rem = axonal remyelination; Fib = fibrosis; $\mathrm{LI}$ = lepromatous infiltrate; MBP = myelin basic protein; MNI = mononuclear infiltrate; ND = not done; NF200 = neurofilament 200 protein; NGFr = nerve growth factor receptor; PCR = polymerase chain reaction for Mycobacterium leprae DNA; PGL-1 = serum antibodies to phenolic glycolipid$1, \mathrm{PGP}=$ protein gene product; Presence of myel fib = presence of myelinated fibers; $\mathrm{TI}=$ tuberculoid infiltrate.

AFB score: $3+=1$ to 10 bacilli per $x 1000$ field, $4+=10$ to 1000 bacilli per $x 1000$ field, $6+=$ more than 1000 bacilli per $x 1000$ field. aScore on semithin sections: presence of myelinated fibers $(0=$ total loss $/ 4=$ normal); presence of axonal degeneration, axonal regeneration, and axonal remyelination $(0=a b s e n t / 4=$ maximal presence $)$. ${ }^{b}$ Score of alteration of immunoreactivity $(0=$ no staining $/ 4=$ maximal staining $)$.

Figure 3. Histopathological examination of semithin sections of inflammatory infiltrate and loss of myelinated fibers. A, Nerve fascicle with a reduced number of myelinated fibers (arrows) and a few inflammatory cells surrounding thickened microvessels (arrowhead) in the endoneurial compartment (end). $B$, Nerve fascicle with complete absence of myelinated fibers and few macrophages surrounding thickened endoneurial microvessels (arrows) in the endoneurial compartment (end). $C$, Endoneurium (end) of a nerve fascicle with a dense inflammatory infiltrate composed of vacuolated macrophages (arrowheads) and lymphocytes surrounding a prominent, branched microvessel (arrow). Note the absence of myelinated fibers. $D$, Nerve fascicle with a reduced number of myelinated fibers and enlarged subperineurial space (sps). Atrophic axons can also be seen (arrows). Scale bar $=40 \mu \mathrm{m}$ for all panels.
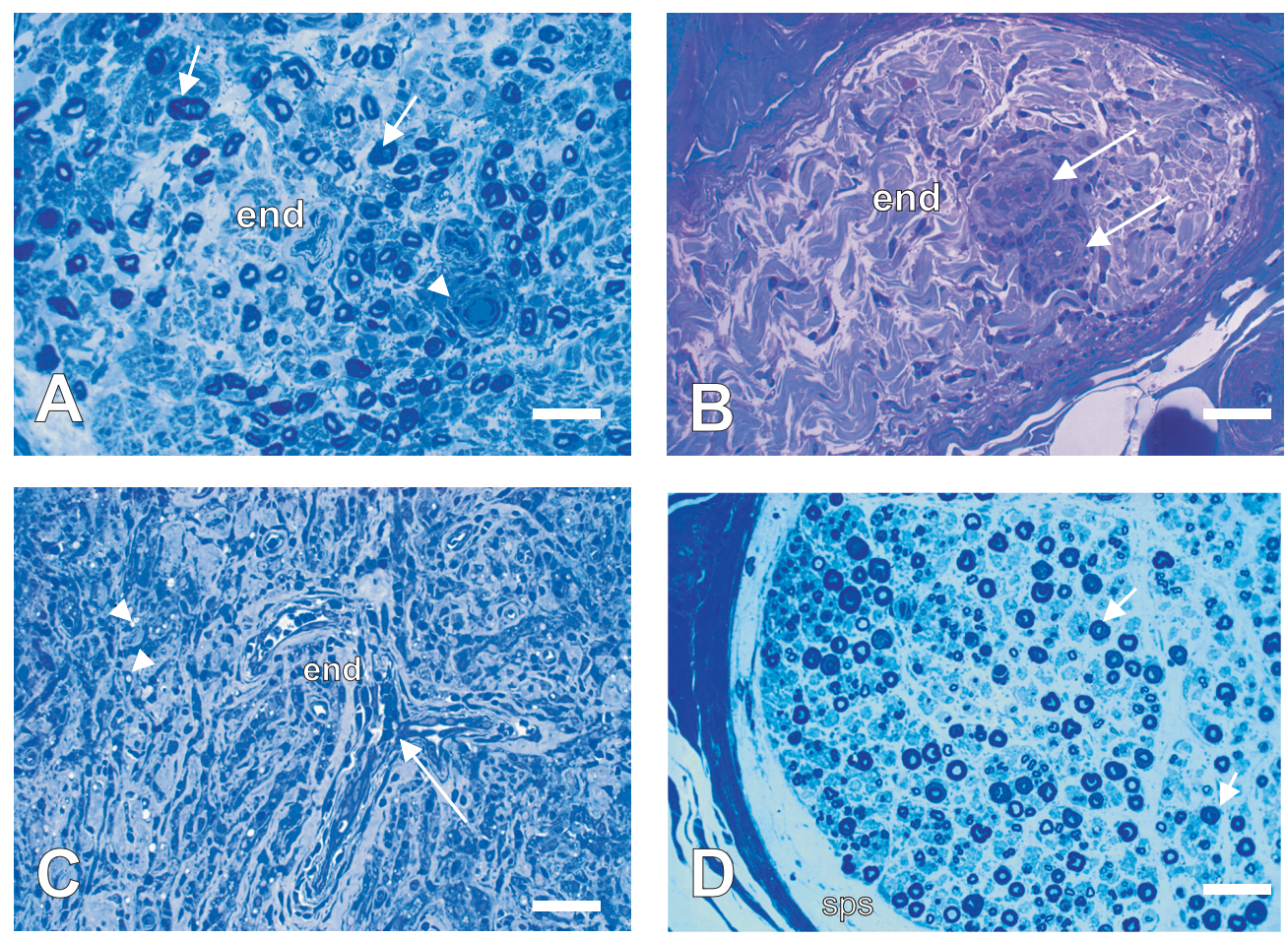
$90.9 \%$ of the nerve biopsies, respectively. After associating the clinical and electroneuromyographic alterations with the immunohistochemistry results, semithin sections of $100 \%$ of the biopsies that showed decreased potential amplitudes also exhibited myelinated fiber loss (Table 3). Another association was seen between reduced potential amplitudes and a lower number of NF200-stained (45.4\%) and PGP 9.5-stained (63.6\%) fibers. In $100 \%$ of the biopsies, the loss of myelinated fibers observed on semithin sections was associated with decreased MBP immunostaining. Moreover, in 40.0\% of the biopsies, small fibers, represented by immunoreactive dots, were seen amid the remaining inflammatory cells even after the larger myelinated fibers had disappeared.

\section{Discussion}

Reflecting the characteristic nerve damage and nerve function impairment occurring in this disease, the expression of neural markers on the leprosy-affected nerves was considerably modified. The number of myelinated fibers with NF200 immunoreactivity was decreased due to the loss of myelinated fibers observed on semithin sections. However, there was no noticeable decrease in the staining intensity of individual fibers. NF200 staining was also detected on the small fibers amid inflammatory cells. Several hypotheses have been raised to explain the progressive axonal degeneration charac- teristic of leprosy, and NGF deprivation is certainly one of them. According to Anand (22), the absence of this target-derived cytokine could be implicated in the pathogenesis of axonal degeneration. Kumar and Sengupta (23) also found basement membrane multilayering of endoneurial microvessels in addition to endothelial hypertrophy, possibly resulting in microvessel obstruction and local ischemia of the nerve.

Sc S-100 protein staining of myelinated fibers was also reduced due to the aforementioned fiber loss. The finding of S100-positive and PGP-negative nerve fibers in the endoneurial compartment could be attributed to the presence of denervated Sc resulting from progressive axonal degeneration and concomitant Sc survival. This observation supported the detection by Shetty and Antia (24) of axon-free Sc in the advanced leprosy neuritic process during which the collagen fibers are surrounded by Sc cytoplasmic protrusions (collagen pockets). These surviving cells might also function as guides in axonal regeneration, which, however, does not seem to be entirely effective in leprosy neuropathy (25). The survival of denervated $\mathrm{Sc}$ is mediated by the production of autocrine cytokines supporting resistance of Sc to death signals and influencing the neuritic process (1). Oliveira et al. (26) have demonstrated the presence of apoptotic Sc in the dermal nerve branches of cutaneous leprosy lesions in addition to demonstrating that synthetic $M$. leprae lipids could activate

Table 3. Association of patients' clinical symptoms with immunohistochemistry findings.

Association of clinical symptoms with immunohistochemistry findings

Reduced potential amplitudes and loss of myelinated fibers

Reduced potential amplitudes and reduced NF200

Reduced potential amplitudes and reduced PGP staining

Hypoesthesia and reduced NGFr staining of small fibers

Hypoesthesia and reduced PGP staining

Loss of myelinated fibers in semithin sections and reduced MBP staining

MBP = myelin basic protein; NF200 = neurofilament 200 protein; NGFr = nerve growth factor receptor; PGP = protein gene product.

Percentage of biopsies
100

45.4

63.6

81.8

90.9

100 
apoptosis in the transformed ST88-14 Sc line through Toll-like receptor 2 molecules. The secreted survival factors might possibly neutralize the Toll-like receptor 2 moleculesmediated death signal.

NGFr staining of neuritic nerves was reduced compared to control and was restricted to the Sc and/or axons of small fibers. It was impossible, however, to distinguish axonal from Sc immunoreactivity in the small fiber profiles since light microscopy does not permit this type of distinction. The staining of MBP was also decreased because of the reduced number of myelinated fibers. The MBP immunopositivity of some endoneurial cells not accompanied by axonal marker staining (data not shown) might represent the myelin debris of degenerated axons.

Axonal degeneration could also be a consequence of demyelination, which might be induced by the presence of $M$. leprae. The cause of demyelination in leprosy is controversial with two divergent hypotheses having been raised in the literature. One explanation refers to M. leprae-induced demyelination and the other to immune-mediated demyelination. In regard to the first, Rambukkana et al. (12) have demonstrated a direct demyelinating effect of $M$. leprae on Sc neuron co-cultures, in agreement with the hypothesis that $M$. leprae has a direct effect on nerve fibers. Vardhini et al. (27) have pointed out the molecular mimicry enacted by mycobacterial proteins (ferredoxinNADP-reductase and a conserved mycobacterial membrane protein) that could mimic $\mathrm{P} 0$, a protein that aids in compacting myelin through homotypical interactions. Molecular mimicry could disrupt tidy myelin layers, with the consequent occurrence of demyelination. It is known that myelin-axon interactions are crucial for the maintenance of axon trophism and function inasmuch as axonal degeneration is usually associated with demyelination. Regarding the second explanation proposed by Spierings et al. (10), an immunopathogenetic demyelination mechanism in which cytotoxic T-cells could be responsible for killing antigen-presenting Sc is the causative factor of demyelination.

In the present study, PGP 9.5 staining was predominantly found on small fibers in the form of clusters of small positive dots attached to Sc in the transverse histological sections of the fascicles. The presence of endoneurial MBP negativity together with PGP and neurofilament-positive clustered dots in the cytoplasm of $\mathrm{Sc}$ on adjacent sections strongly suggested that these dots were immunoreactive, non-myelinated axons.

It is interesting to note that, despite the assumption found in the literature that leprosy predominantly affects small nerve fibers (12), these fibers were accompanied by the complete disappearance of myelinated fibers and MBP immunoreactivity and could still be seen among the inflammatory cells after staining of the PGP and NF200 axonal markers. As a result, small fibers (including non-myelinated ones) might actually be less sensitive to the environmental endoneurial disturbances taking place during the course of neuritic leprosy. It is recommended that an in-depth morphological assessment of the status of small fibers be conducted using electron microscopy.

Sensory impairment (thermal, algic, and tactile hypoesthesia) associated with small fiber disturbances in the endoneurium suggested that either the number of fibers had been reduced or that at the time of the biopsy these fibers, normally involved in nociceptive sensation, were undergoing functional alterations. Antunes et al. (28) also raised this last hypothesis when they found no significant quantitative difference between the number of fibers in biopsies taken during or after reactional episodes. It is worth noting that the lack of immunoreactivity of a neural marker in the endoneurium does not necessarily mean the absence of fibers. It might reflect a qualitative modification within the nerve fiber, which may also be accompanied 
by a functional disturbance. Again, the quantification and morphological status of small non-myelinated fibers are more adequately assessed by electron microscopy studies.

The reduced potential amplitudes found in electroneuromyography were associated with the axonal loss shown by NF, PGP 9.5, and the corresponding semithin section analysis. Moreover, in the electroneuromyographic study, the presence of remyelinating axons on the semithin sections was accompanied by an electroneurographic demyelination pattern in only two cases. This may have occurred because, as demonstrated by Jacobs et al. (11), the morphological signs of active demyelination in the nerves affected by leprosy via the teasing technique did not seem to be as dramatic as that which commonly occurs in both chronic inflammatory demyelinating neuropathy and genetic my- elination disorders. Furthermore, in leprosy neuropathy, the onset of axonal degeneration due to the neuritic process may be masking the appearance of a fully developed demyelinating process.

Therefore, leprosy is the cause of highly destructive peripheral neuritis as evidenced by the reduced immunohistochemical expression of neural markers and loss of myelinated fibers on the semithin sections. These findings were associated with the clinical and electroneuromyographical manifestations of peripheral neuropathy shown by leprosy patients.

\section{Acknowledgments}

We thank Judy Grevan for reviewing the English and editing the text.

\section{References}

1. Fite GL. The pathology and pathogenesis of leprosy. Ann N Y Acad Sci 1951; 54: 28-33.

2. Ustianowski AP, Lockwood DN. Leprosy: current diagnostic and treatment approaches. Curr Opin Infect Dis 2003; 16: 421-427.

3. Girdhar BK. Neuritic leprosy. Indian J Lepr 1996; 68: 35-42.

4. Talwar S, Jha PK, Tiwari VD. Neuritic leprosy: epidemiology and therapeutic responsiveness. Lepr Rev 1992; 63: 263-268.

5. Pannikar VK, Arunthathi S, Chacko CJ, Fritschi EP. A clinico-pathological study of primary neuritic leprosy. Lepr India 1983; 55: 212221.

6. Jardim MR, Antunes SL, Santos AR, Nascimento OJ, Nery JA, Sales AM, et al. Criteria for diagnosis of pure neural leprosy. $J$ Neurol 2003; 250: 806-809.

7. Bryceson A, Pfaltzgraff RE. Complications due to nerve damage. In: Hastings RC (Editor), Leprosy. Medicine in the tropics. 3rd edn. Edinburgh: Churchill-Livingstone; 1990.

8. Chimelli L, Freitas M, Nascimento O. Value of nerve biopsy in the diagnosis and follow-up of leprosy: the role of vascular lesions and usefulness of nerve studies in the detection of persistent bacilli. $J$ Neurol 1997; 244: 318-323.

9. Dastur DK, Pandya SS, Antia NH. Nerves in the arm in leprosy. 2. Pathology, pathogenesis and clinical correlations. Int $J$ Lepr Other Mycobact Dis 1970; 38: 30-48.

10. Spierings E, de Boer T, Wieles B, Adams LB, Marani E, Ottenhoff TH. Mycobacterium leprae-specific, HLA class II-restricted killing of human Schwann cells by CD4+ Th1 cells: a novel immunopathogenic mechanism of nerve damage in leprosy. $J$ Immunol 2001; 166 : 5883-5888.
11. Jacobs JM, Shetty VP, Antia NH. Teased fibre studies in leprous neuropathy. J Neurol Sci 1987; 79: 301-313.

12. Rambukkana A, Zanazzi G, Tapinos N, Salzer JL. Contact-dependent demyelination by Mycobacterium leprae in the absence of immune cells. Science 2002; 296: 927-931.

13. Franke FE, Schachenmayr W, Osborn M, Altmannsberger M. Unexpected immunoreactivities of intermediate filament antibodies in human brain and brain tumors. Am J Pathol 1991; 139: 67-79.

14. Lowe J, McDermott H, Landon M, Mayer RJ, Wilkinson KD. Ubiquitin carboxyl-terminal hydrolase (PGP 9.5) is selectively present in ubiquitinated inclusion bodies characteristic of human neurodegenerative diseases. J Pathol 1990; 161: 153-160.

15. Antunes SL, Sarno EN, Holmkvist G, Johansson O. A comparison of the expression of NGFr, PGP 9.5 and NSE in cutaneous lesions of patients with early leprosy using immunohistochemistry. Int $J$ Lepr Other Mycobact Dis 1997; 65: 357-365.

16. Chesa PG, Rettig WJ, Thomson TM, Old LJ, Melamed MR. Immunohistochemical analysis of nerve growth factor receptor expression in normal and malignant human tissues. J Histochem Cytochem 1988; 36: 383-389.

17. Deibler GE, Martenson RE, Kies MW. Large scale preparation of myelin basic protein from central nervous tissue of several mammalian species. Prep Biochem 1972; 2: 139-165.

18. Carson FL, Martin JH, Lynn JA. Formalin fixation for electron microscopy: a re-evaluation. Am J Clin Pathol 1973; 59: 365-373.

19. Jardim MR, Antunes SL, Simons B, Wildenbeest JG, Nery JA, Illarramendi $X$, et al. Role of PGL-I antibody detection in the diagnosis of pure neural leprosy. Lepr Rev 2005; 76: 232-240. 
20. Santos AR, De Miranda AB, Sarno EN, Suffys PN, Degrave WM. Use of PCR-mediated amplification of Mycobacterium leprae DNA in different types of clinical samples for the diagnosis of leprosy. $J$ Med Microbiol 1993; 39: 298-304.

21. Hayat MA. Principles and techniques of electron microscopy. Vol. 1. Biological applications. New York: Van Nostrand Reinhold Company; 1981.

22. Anand $P$. Neurotrophic factors and their receptors in human sensory neuropathies. Prog Brain Res 2004; 146: 477-492.

23. Kumar V, Sengupta U. Ultrastructural study of Schwann cells and endothelial cells in the pathogenesis of leprous neuropathy. Int $J$ Lepr Other Mycobact Dis 2003; 71: 328-340.

24. Shetty VP, Antia NH. Pathology of nerve damage in leprosy. In: Antia NH, Shetty VP (Editors), The peripheral nerve in leprosy and other neuropathies. Calcutta: Oxford India Publication; 1997. p 79137.
25. Miko TL, Gschmeissner SE, le Maitre C, Kinfu Y, Kazen R, Pereira $\mathrm{JH}$. Regeneration at the predilective damage sites of nerve trunks in treated leprosy. Lepr Rev 1993; 64: 330-337.

26. Oliveira RB, Ochoa MT, Sieling PA, Rea TH, Rambukkana A, Sarno EN, et al. Expression of Toll-like receptor 2 on human Schwann cells: a mechanism of nerve damage in leprosy. Infect Immun 2003; 71: 1427-1433.

27. Vardhini D, Suneetha S, Ahmed N, Joshi DS, Karuna S, Magee X, et al. Comparative proteomics of the Mycobacterium leprae binding protein myelin P0: its implication in leprosy and other neurodegenerative diseases. Infect Genet Evol 2004; 4: 21-28.

28. Antunes SL, Liang $Y$, Neri JA, Haak-Frendscho M, Johansson $O$. The expression of NGFr and PGP 9.5 in leprosy reactional cutaneous lesions: an assessment of the nerve fiber status using immunostaining. Arq Neuropsiquiatr 2003; 61: 346-352. 